\title{
Application of Biotechnology in Organic Waste Gas Treatment
}

\author{
Zun Dai ${ }^{1, a}$ \\ ${ }^{1}$ College of Agriculture, Hainan University, Haikou, Hainan, China, 570228 \\ a email
}

Keywords: Application, Biotechnology, Organic Waste Gas Treatment

\begin{abstract}
Biotechnology purification organic waste gas is a new emission control technology gradually developed in recent years, with features of high efficiency, low investment and operating costs, no secondary pollution, safety and good. In this paper, the concept of bio-organic waste gas treatment technology, mechanism, characteristics and research development of the system were discussed and we proposed the existing problems and future research directions.
\end{abstract}

\section{Introduction}

With the rapid development of synthetic organic industrial and petrochemical industries, the volatile organic compounds into the atmosphere are increasing. These substances mainly as hydrogen sulfide, ammonia, amines, nitro based compounds, hydrocarbons, fatty acids, alcohols, phenols, esters and organic halogen derivatives, etc. They often with stench, not only for humans and a variety of sensory stimulation, and has some toxicity, some will have a "three-induced" effect on the human body and the environment great harm.

Currently VOC treatment methods are non-destructive method, destructive methods and joint method both of which less than conventional biological treatment technology investment, low operating costs, simple operation, wide range of applications, is the most promising alternative to combustion and new technologies adsorption purification method for VOC biological treatment research reports first appeared in the United States, while the more widely studied abroad from the 1970s began, the country began in related research the early 1990s. These studies include the basic principles and methods, devices, equipment and operating conditions of the biological treatment of organic waste, organic waste capable of degrading microbial populations and their conditions of formation on the packing, the dynamic model. The main purpose of research and analysis is to remove odor, process the mixture of a variety of low concentrations of volatile organic compounds present, foreign countries have been on a small scale, pilot production and research, and has been successful as an engineering application; while domestic the research is still at bench scale.

\section{The Basic Concepts of Biological Purification of Organic Waste}

Biological purification of organic waste is developed has been proven microbial wastewater treatment basis. Biological purification is essentially a process of oxidation and decomposition: active microorganisms adhered on the porous, moist media to the organic component in the exhaust gas as their energy or life activity of nutrients into simple inorganic substances (CO2, H2O) or cells substance.

The biggest difference between biological wastewater treatment process is that: organic matter in the exhaust gas from the gas phase to the first through the liquid (or solid surface film) mass transfer process, and then dissolved in the liquid phase of the organic component at a concentration of poor push to further spread the surrounding medium, and then absorb the microorganisms captured; in this condition, into the body of contaminant microorganisms as an energy source and nutrients are decomposed in their own metabolism, metabolites produced by dissolving a portion into the liquid phase, as a part of the energy metabolism of cells or cellular material, and part (e.g., CO2) is precipitated into the air. Organic matter in the exhaust gas through the above process is declining, so as to be purified. 


\section{The Principle of Organic Waste Biological Treatment}

Organic waste biological treatment process is essentially the use of microbial life activities will change the exhaust of harmful substances into simple inorganic substances (such as $\mathrm{CO} 2$ and $\mathrm{H} 2 \mathrm{O}$ ) and cell substances. Mechanism of biochemical processing of organic waste is still no unified theory, internationally accepted Dutch scholar Ottengraf is based on the traditional gas absorption two-film theory - the theory according to biofilm theory, biochemical processing of organic waste in general go through three steps: 1) organic pollutants in the exhaust gas first contact with water and dissolved in water (that is, from the air membrane diffusion into the film); 2) dissolved in a liquid film organic component at a concentration of poor push further spread to the biofilm, which in turn is a microorganism to capture and absorb; 3) organic pollutants into the microorganisms as energy and nutrients are broken down in its own metabolism, the biochemical reactions eventually transformed into harmless compounds.

\section{The Technological Features of Biology}

Since the microorganisms of various pollutants have a strong, fast adaptability, and as a metabolic substrate and its degradation, transformation. Therefore, as compared with the conventional exhaust gas treatment technologies, biological treatment technology has good effect, low investment and operating costs, safety, and no secondary pollution, easy to manage and so on. Meanwhile, since the exhaust gas processing biological regeneration of the absorbent can be used directly by the action of microorganisms in the absorbent achieved without having to determine physical and chemical adsorption and absorption as specialized equipment, thereby simplifying the process and the industrial equipment reducing the cost of running the operation. Therefore, biological treatment technology has become one of the hot topics in the world of research.

\section{The Main Biological Treatment Technology}

Biological Filter Bed. It is a biological filter bed in which filled with adsorption media (such as peat, soil, activated carbon and other substances) purification device. Before hanging biofilm, $\mathrm{pH}$ buffering agents and the incorporation of N, P, K and other nutrients (such as NH4NO3 and K2HPO3) in the filter bed, when the exhaust gas has a certain moisture into the biological filter bed by about 0.5 - $1 \mathrm{~m}$ thick biological activity when bed materials in the media of microorganisms (mainly bacteria, protozoa, algae, etc.) can be captured by contacting the organic matter and the exhaust gas as a carbon source for growth itself. Thus, after the exhaust gas passes through the biological filter bed can be purified and the filter layer microbial biodegradation of pollutants in the process of continuous growth and reproduction, so that the biofilter operation to continue. Media after one year is generally acidic, to regular maintenance and upkeep.

Biological treatment of sewage with different filter bed, in the biological treatment of exhaust gas in the filter bed, but stagnant water on the inner surface and the biofilm is not formed throughout the filter bed continuous flow phase, it may be an aqueous biofilms regarded as a single phase, or so-called solid / liquid mixed phase. An exhaust gas purification process in a biological filter bed mass may be considered as a series of biochemical reactions. Qualitatively, while the mass transfer of gaseous pollutants direction is transmitted to the mixed phase solid / liquid. Is generally believed that the biochemical reaction rate is faster than the rate of mass transfer, it is to control the biochemical reaction step of the process.

Meanwhile, since the filter (particularly activated carbon) having a high adsorption capacity, it can make microbial extracellular enzymes and microbial contaminants and the membrane filter and concentrated at the interface, thereby increasing the rate of biochemical reactions, and the degree of purification of exhaust gas can be improve. Thus, the biological filter bed in the treatment of exhaust gas has been more widely used.

Bioscrubber Bed. Biological washing bed usually consists of a scrubber and a regeneration pool. In the scrubber, the circulating liquid by spraying or bubbling way exhaust gas pollutants and oxygen into 
the liquid phase, to achieve mass transfer. In general, if air resistance is large the spray method is available, on the contrary, when the resistance is large with a liquid bubbling. This process, the total transfer rate of absorption of pollutants and the exhaust gas, the contact area of the spray liquid and the average driving pressure (the difference between the actual concentration of the average concentration of gaseous and liquid waste in the waste) related. Absorbing the washing liquid gas component flows into the regeneration tank (activated sludge tank). In the regeneration pool, pollutants are broken down microbial oxidation, the process is actually microbial treatment process wastewater and wastewater treatment is one of the most mature technologies.

Biological washing tower in the exhaust gas treatment process, due to the absorption and oxidation are two of the process is relatively independent, and therefore easy to control, removal of pollutants in the exhaust gas is high. But the establishment of two separate systems, we need aeration facilities, which resulted in a larger area of the process, high energy consumption.

Biological Trickling Filter Bed. Biological trickling filter bed of coarse gravel, plastic honeycomb filler, plastic packing corrugated board, ceramic, stainless steel rings, bark, carbon fiber, silica gel, and other similar non-absorbent filler, filler surface is microbial biofilm formation of a few millimeters thick. Exhaust gas from the bottom of the bed into the trickling filter, return water to the upper spray from the upper packed bed, and the biofilm on the packing along trickle down, dissolved in water and organic pollutants in the form of biofilms attached to the filler microorganisms absorption, microbial cells into the organic contaminants in microbial metabolic processes in the body as an energy source and nutrients are broken down, eventually transformed into harmless compounds (such as CO2 and $\mathrm{H} 2 \mathrm{O}$ ). The specific surface area of the filler is generally $100 \sim 300 \mathrm{~m} 2$ / $\mathrm{m} 3$, this aspect of the gas by providing a lot of space, on the other hand the gas pressure and space filler layer caused by the clogging due to microbial growth down to a minimum the risk. The reaction conditions for bio-trickling filter bed can easily be controlled by adjusting the $\mathrm{pH}$ of the circulating fluid, adding K2HPO4, NH4NO3 and other substances can be achieved.

Biological trickling filtration bed of filler surface high concentration of microorganisms, growth and stability, there is a continuous flow of water trickling phase in bed, so the whole process involved in the mass transfer of gas, liquid and solid. But overall speaking, the process is still a tandem mass transfer and biochemical reactions. If properly designed to have a high concentration of microorganisms, better resistance to impact load, clean, fast response, gas residence time is short and other advantages, this method has a small reactor volume, equipment investment and low cost, better development and application prospect, is currently one of the hottest countries in biological research of air pollutants treatment.

\section{Processes and Equipment of Organic Waste Biological Treatment}

Processing methods can be divided into biological washing method (suspended state) and biological filtration (fixing state) based on organic waste gas processing microorganisms present in the form of biological washing method (also known as bio-absorption method) that is micro-organisms and nutrients Ingredients present in the liquid, organics from gases by contact with the suspension is transferred into the liquid microbial degradation of biological filtration is the growth of microorganisms attached to a solid medium (filler), the exhaust gas through a fixed bed of dielectric layer composed of (filler layer) is adsorption, absorption, microbial degradation ultimately, more typical biological filter and biological trickling filter two forms.

Bio-Scrubber. Bio-scrubber is a suspended activated sludge treatment system comprising an absorber chamber and a regeneration pool constitute its process shown in Figure 1. The organism suspension (circulating fluid) from the top of the spray absorption chamber and the next, so that the exhaust gas pollution was transferred to the liquid phase, to achieve mass transfer process. Absorb organic matter in the exhaust gas flowing into the suspension of biological regeneration reactor, passing air oxygenation regeneration is absorbed by microbial oxidation of organic matter, eventually renewable biomass pool the 
suspension was removed from the liquid phase. the most commonly used biological activated sludge suspension is a suspension, but a longer time of its regeneration after treatment, a few hours due to the absorption and the different time needed for regeneration, and therefore, a suspension of biological regeneration is in the same reactor, or in another reactor to complete, depending on the strength of activity and biological regeneration capacity of the suspension. Scrubber gas-liquid contacting method in addition to the liquid spray method, but also by gas bubbling, it depends on what is controlling the absorption process steps.

Biofilter. Biofilter treatment of organic waste gas has a moisture process shown in Figure 2. The organic waste into the biofilter, through biological active filler layer $50 \sim 100 \mathrm{~cm}$ and the transfer of pollutants from the gas phase to the biological phase and then be broken down microbial oxidation. Decomposition process is completed by the heterotrophic aerobic microbial neutral and slightly alkaline conditions in general for the final decomposition of organic matter CO2. First organic nitrogen is converted to NH3, and then converted to nitrate; sulfide is first converted to H2S, followed by oxidation of sulfate. Purified gas is discharged.

Filter Material. Biofilter filler layer is adsorptive media, multi-mixed soil, compost, wood chips, charcoal or several media. Media must have good air permeability and moderate through water and water holding capacity, and rich microbial communities act as a filter microorganisms (mainly bacteria and fungi) carrier, which in turn wet microbial biofilm filler particles surrounded. Microbial filter also to provide essential nutrients their lives, these nutrients can be recycled, but eventually it will be broken mineralization. typically, after a few years of running the nutrients are depleted, the need to replace the filter size of the filter particles, generally only provide reasonable adsorption surface, and having a satisfactory sexual stagnation appropriate. Biofilter single filter bed open, multi filter bed enclosed form. Compared with the open single filter bed biofilter, biological filter for closed-end low maintenance requirements and it occupies less affected by climate small and easy to monitor, but higher cost.

\section{Conclusion}

The treatment of wastewater more than 100 years of history by biochemical methods, but the historical process of the exhaust gas is very short, in recent years, with the increasing variety of industrial emissions, and the people gradually improve the living environment of the requirements, exhaust gas pollution control has been more and more people's attention. In view of this situation is gradually research and development of biological treatment of exhaust gas. Now single-component gas, the treatment effect has been good, there are still some problems may be in the actual operation of the process, it has attracted wide attention at home and abroad. Our emissions of laws and regulations is lagging behind, but with the strengthening of air pollution and increase the national management measures, limits on emissions will become increasingly stringent, and therefore, the exhaust gas technology in China's chemical and petrochemical industries as well as paint and other governance has good prospects.

\section{References}

[1] Jia Xinzhang, Li Jingyuan. Environmental Herald, Vol. 6 (2014) No 53, p.25-26

[2] Wang Yunhui, Wang Qunyong. Shanghai Environmental Sciences, Vol. 12 (2015) No 27, p.74-76

[3] Qian Xiyuan, Jing Jianfen. Sichuan Environment, Vol. 30 (2014) No 19, p.144-145

[4] Wang Kuailiang. Chemical Environmental Protection, Vol. 29 (2011) No 27, p.21-23

[5] Zhang Gongxu, Sun Jing. Environmental Progress, Vol. 8 (2013) No 27, p.57-60 